\title{
Fatores associados à não adesão ao uso de anticoagulantes orais: protocolo de revisão sistemática
}

\author{
Factors associated with non-adherence to the use of oral anticoagulants: a systematic review protocol \\ Factores asociados a la no adherencia al uso de anticoagulantes orales: un protocolo de revisión sistemática
}

\begin{abstract}
RESUMO
Objetivo: Descrever fatores associados à não adesão ao uso de derivados cumarínicos e anticoagulantes orais de ação direta. Método: Serão elegiveis os estudos observacionais e experimentais, com pacientes $\geq 18$ anos em anticoagulação oral. A busca de artigos será nas bases MEDLINE (via PubMed), EMBASE, CINAHL e Literatura Latino-Americana e do Caribe, além de busca manual nas listas de referência dos estudos selecionados. As duplicatas serão removidas. Será feita leitura do título/resumo dos artigos para pré-seleção daqueles que serão lidos na íntegra para definir sobre sua inclusão na revisão. As informações serão extraídas, tabuladas e descritas de forma narrativa. Resultado: Na busca preliminar foram encontrados 1270 estudos, sendo selecionados 21 artigos para a revisão sistemática após realização das etapas metodológicas. Conclusão: 0 conhecimento dos fatores associados à não adesão à anticoagulação oral pode melhorar o entendimento sobre aspectos do tratamento. Cadastro de registro da revisão sistemática (PROSPERO): CRD 42020223555.
\end{abstract}

DESCRITORES: Anticoagulantes; Cumarínicos; Inibidores do fator Xa; Revisão sistemática; Tromboembolismo

\section{ABSTRACT}

Objective: To describe factors associated with non-adherence to the use of coumarin derivatives and direct acting oral anticoagulants. Method: Observational and experimental studies with patients $\geq 18$ years on oral anticoagulation will be eligible. The search for articles will be conducted in MEDLINE (via PubMed), EMBASE, CINAHL and Latin American and Caribbean Literature, as well as a manual search of the reference lists of the selected studies. Duplicates will be removed. The title/summary of the articles will be read to pre-select those that will be read in full to define their inclusion in the review. The information will be extracted, tabulated and described in narrative form. Result: In the preliminary search 1270 studies were found, and 21 articles were selected for the systematic review after the methodological steps. Conclusion: Knowledge of factors associated with non-adherence to oral anticoagulation may improve understanding of treatment aspects. Systematic review registration (PROSPERO): CRD 42020223555.

DESCRIPTORS: Anticoagulants; Coumarins; Factor Xa inhibitors; Systematic review; Thromboembolism

\section{RESUMEN}

Objetivo: Describir los factores asociados a la falta de adherencia al uso de derivados cumarínicos y anticoagulantes orales de acción directa. Método: Serán elegibles los estudios observacionales y experimentales con pacientes $\geq 18$ años en anticoagulación oral. La búsqueda de artículos se realizará en las bases de datos MEDLINE (a través de PubMed), EMBASE, CINAHL y Literatura Latinoamericana y del Caribe, además de una búsqueda manual en las listas de referencias de los estudios seleccionados. Se eliminarán los duplicados. Se leerá el título/resumen de los artículos para preseleccionar los que se leerán en su totalidad para definir su inclusión en la revisión. La información se extraerá, se tabulará y se describirá en forma de relato. Resultados: En la búsqueda preliminar se encontraron 1270 estudios, siendo seleccionados 21 artículos para la revisión sistemática tras realizar los pasos metodológicos. Conclusión: El conocimiento de los factores asociados a la no adherencia a la anticoagulación oral puede mejorar la comprensión de los aspectos del tratamiento. Registro de revisión sistemática (PROSPERO): CRD 42020223555. DESCRIPTORES: Anticoagulantes; Cumarina; Inhibidores del factor Xa; Revisión sistemática; Tromboembolismo

RECEBIMENTO: 17/05/2021 APROVAÇÃO: 18/08/2021 


\title{
Waleska Jaclyn Freitas Nunes de Sousa
}

Graduação completa em farmácia. Mestrado incompleto em Ciências de Saúde. Hospital das Clínicas da Universidade Federal de Minas Gerais.

ORCID: 0000-0002-6454-7983

\section{Nathália Sernizon Guimarães}

Doutora. Faculdade de Medicina da Universidade Federal de Minas Gerais.

ORCID: 0000-0002-0487-0500

\section{Catiane Costa Viana}

Mestre. Faculdade de Farmácia, Universidade Federal de Minas Gerais.

ORCID: 0000-0001-6237-8541

\section{Amanda Fonseca Medeiros}

Bacharel em farmácia. . Faculdade de Farmácia, Universidade Federal de Minas Gerais

ORCID: 0000-0002-6747-6172

\author{
Mayara Sousa Vianna \\ Enfermeira. Faculdade de Farmácia, Universidade Federal de Minas Gerais \\ ORCID: 0000-0003-1427-2149
}

\section{Caryne Margotto Bertollo}

Hospital das Clínicas da Universidade Federal de Minas Gerais. Faculdade de Farmácia, Universidade Federal de Minas Gerais ORCID: 0000-0003-1427-2149

\section{INTRODUÇÃO}

Os anticoagulantes orais são usados para prevenção primária e secundária de tromboembolismo em indivíduos com fatores de risco como, por exemplo, a fibrilação atrial (FA) 1,2. Em pessoas com diagnóstico de FA existe risco aumentado de desenvolvimento de acidente vascular cerebral (AVC)3, que é a terceira principal causa de morte em todo o mundo4. Apesar dos benefícios da anticoagulação oral, alguns pacientes são mais propensos a desenvolver eventos adversos a medicamentos, representados principalmente por tromboembolismo e sangramento, que estão relacionados à falha terapêutica e efeito anticoagulante excessivo, respectivamente5.

Os derivados cumarínicos têm sido utilizados há décadas como anticoagulantes orais1,6,7. Para algumas indicações, esses fármacos têm sido substituídos, gradativamente, por anticoagulantes orais de ação direta, sendo que ambas as opções terapêuticas são eficazes na prevenção de AVC6,7,8,9,10,11,12. Porém, os anticoagulantes orais de ação direta têm limitações de uso em pacientes com próteses cardíacas mecânicas, insuficiência renal grave e FA com doença cardíaca reumática associada a estenose mitral grave. Além disso, faltam evidências para apoiar o uso de anticoagulantes orais de ação direta em pessoas com disfunção hepática moderada e grave 13 .

A varfarina é um derivado cumarínico amplamente utilizado com benefícios reconhecidos, embora apresente como desvantagens o índice terapêutico estreito 7 , ampla variabilidade dose-resposta14, potencial para interagir com plantas medicinais 15 , vários medicamentos6,16,17 e alimentos com alto teor de vitamina K6. O monitoramento da Razão Normalizada Internacional (RNI), obtido a partir do tempo de protrombina, é necessário para orientar os ajustes de dose da varfarina e aumentar a segurança na utilização do medicamento 18 .

Os anticoagulantes orais de ação direta são representados por inibidor direto da trombina (dabigatrana) e inibidores do fator Xa (edoxabana, rivaroxabana e apixabana) 19,20,21,22. Esses, ao contrário da varfarina, não requerem ajuste de dose individualizado6 e apresentam menos interações com alimentos e outros medicamentos23. No entanto, o alto custo se apresenta como uma desvantagem 24 e a meia-vida mais curta em comparação aos derivados cumarínicos pode aumentar o risco de eventos tromboembólicos em pacientes com baixa adesão ao tratamento20.

A obtenção de um controle ideal da anticoagulação oral é um desafio na prática clínica e a adesão é crucial para o sucesso do tratamento25,26,27. Segundo a Organização Mundial da Saúde (2003), o conceito de adesão diz respeito à administração do medicamento pelo paciente e à adoção de comportamentos, como mudanças no estilo de vida e dieta de acordo com recomendações baseadas em evidências passadas pelos profissionais responsáveis pelo cuidado ao paciente. Desse modo, principalmente para aqueles que necessitam de tratamentos prolongados, a não adesão torna-se um problema importante a ser considerado2 8 e que precisa ser abordado para assegurar efetividade e segurança da anticoagulação oral.

Para mensurar a adesão, podem ser utilizados métodos diretos e indiretos. Os métodos diretos compreendem medidas da concentração do fármaco ou metabólitos em fluidos como sangue e urina ou de marcadores biológicos no sangue29. Os métodos indiretos englobam dispositivos de monitoramento eletrônico, avaliação da resposta clínica, contagem de compri- 
midos, aplicação de questionários e autorrelato de adesão ao tratamento. Esse último traz as vantagens de ser simples, de baixo custo e de grande utilidade no cenário clínico pela sua facilidade de aplicação. Ambos os métodos têm desvantagens, sendo que os diretos apresentam alto custo e os indiretos possuem a possibilidade de superestimar a adesão quando se realiza entrevista com o próprio paciente 30.

O conhecimento sobre os fatores associados à não adesão ao tratamento com anticoagulantes orais poderá melhorar o entendimento sobre aspectos do processo assistencial voltado a esse perfil de pacientes. Desse modo, conhecer esses fatores pode ser útil para a prática clínica de farmacêuticos e outros profissionais para fundamentar intervenções visando melhoria na adesão ao medicamento e os resultados do tratamento. Esta revisão tem como objetivo descrever os fatores associados à não adesão à anticoagulação oral com derivados cumarínicos e anticoagulantes orais de ação direta.

\section{MÉTODOS}

Este protocolo será conduzido de acordo com o checklist PRISMA-P31 e se encontra registrado no PROSPERO, sob o código CRD 42020223555.

Os estudos de interesse são aqueles que avaliaram os fatores associados à não adesão ao tratamento com derivados cumarínicos ou anticoagulantes orais de ação direta em pacientes $\geq 18$ anos, de ambos os sexos, em anticoagulação oral para qualquer indicação de tromboprofilaxia. Os métodos diretos e indiretos serão considerados relevantes para obtenção de dados de não adesão.

Estudos observacionais (transversal, caso-controle e coorte) e experimentais em humanos (ensaios clínicos) serão avaliados para elegibilidade. Não haverá restrição de idioma ou de data de publicação.

Serão excluídas as revisões sistemáticas com ou sem meta-análise, revisões integrativas ou narrativas, séries de casos ou relatos de casos e estudos experimentais com animais.

Para melhorar a sensibilidade das estra- tégias de pesquisa, serão utilizados termos citados pelo Medical Subject Heading $(\mathrm{MeSH})$, bem como termos não $\mathrm{MeSH}$, caracterizados como jargões da prática clínica. A estratégia de busca inicial será adaptada e replicada para cada base de dados usando palavras-chave e termos indexados relacionados ao tema de interesse, compreendendo Emtree para Embase e DeCS para Literatura Latino-Americana e do Caribe (LILACS). Os termos indexados foram definidos para compor as estratégias de busca em abril de 2021 (Apêndice A).

Seguindo a estratégia PICO (P: pacientes; I: intervenção; C: controle; O: desfecho), os domínios definidos para as estratégias de busca serão a população do estudo (pacientes $\geq 18$ anos, ambos os sexos), os métodos utilizados para medir a não adesão à anticoagulação oral (emprego de métodos diretos ou indiretos) e as variáveis de interesse (fatores associados à não adesão à anticoagulação oral) (Apêndice B). O critério de controle não é aplicável para o propósito da revisão.

As bases de dados consideradas para as buscas eletrônicas serão MEDLINE (via PubMed), Embase, CINAHL e LILACS. Além disso, será realizada uma busca manual para encontro de estudos relevantes nas listas de referências dos artigos selecionados. A literatura cinza (Google Scholar, MedNar, OpenGray e ProQuest Dissertações e Teses) também será considerada para a busca de artigos.

Todos os artigos selecionados serão exportados para o programa Rayyan QCRI (Doha, Qatar, 2016)32, onde as duplicatas serão detectadas e removidas antes da triagem para elegibilidade. A seleção dos estudos ocorrerá com a leitura inicial do título e resumo por dois revisores independentes (WJFNS e NGS), seguida da leitura na íntegra dos artigos selecionados nesta etapa por dois revisores (WJFNS e NGS).

Os artigos que não atenderem aos critérios de inclusão após a leitura completa serão excluídos. $\mathrm{O}$ motivo da exclusão dos estudos em cada etapa será apresentado em fluxograma indicado pelo PRISMA Statement: an Updated Guideline for Reporting Systematic Reviews33 (Apêndice C). Um terceiro revisor (MAPM) resolverá eventuais discordâncias que tenham ocorrido nas etapas anteriores, realizadas de forma independente pelos dois revisores.

A extração de dados será realizada por dois revisores (WJFNS e NGS) e as divergências serão resolvidas por meio de discussão com um terceiro revisor (MAPM). Para assegurar que as ferramentas de extração de dados capturem todas as informações necessárias, será feito estudo piloto incluindo três artigos selecionados aleatoriamente até que os dois autores sejam capazes de registrar as informações extraídas sem divergências.

Os estudos selecionados serão lidos e as informações de interesse extraídas da seguinte forma: informaçôes do estudo (título, autor, ano, país, desenho do estudo, número de participantes, método de medida de não adesão (métodos diretos ou métodos indiretos), anticoagulante oral avaliado (derivados cumarínicos e anticoagulantes orais de ação direta), variáveis testadas, tempo de uso avaliado, medidas de associação e fatores associados à não adesão), dados sociodemográficos e comportamentais (idade, sexo masculino, escolaridade, consumo de bebidas alcoólicas e tabagismo), dados clínicos e farmacoterapêuticos (indicação da anticoagulação oral, comorbidades, ocorrência de eventos (tromboembolismo ou hemorragias) e outros medicamentos em uso crônico) (Apêndice D).

Os autores dos estudos serão contatados para solicitar informações faltantes, se necessário. Aqueles estudos sem resposta dos autores correspondentes terão as informações identificadas como "faltantes" na apresentação de resultados.

Os dados serão extraídos e descritos em tabelas. Os fatores associados à não adesão ao tratamento com derivados cumarínicos e anticoagulantes orais de ação direta são as variáveis de interesse e serão discutidos. Os resultados desta revisão sistemática fornecerão dados sobre os fatores associados à não adesão à anticoagulação oral e podem ser úteis para a tomada de decisão na assistência ao paciente.

Os estudos de caso-controle e de coorte terão sua qualidade metodológica avaliada 
por meio de Newcastle-Ottawa Quality Assessment Scale34. Essa lista de verificação contém oito itens, com três domínios em cada um. A pontuação máxima que pode ser obtida é nove pontos. Os escores determinam o nível de qualidade do estudo, com pontos de corte indicando qualidade alta (7-9 pontos), moderada (4-6 pontos) e baixa (0-3 pontos). A Lista de verificação da metodologia da Agency for Research and Health Quality (ARHQ) para estudos transversais/de prevalência35 será usada para avaliar a qualidade metodológica dos estudos transversais. Essa escala possui 11 itens com três domínios ("sim", "não" e "pouco claro") a serem preenchidos e os resultados são pontuados com a resposta "sim". A pontuação final é categorizada como qualidade baixa (0-3 pontos), moderada (4-7 pontos) e alta (8-11 pontos).

A segunda versão da RoB 2 será utilizada para avaliação de estudos experimentais. Essa ferramenta da Cochrane foi validada para verificação da qualidade metodológica dos estudos experimentais e contém cinco domínios de avaliação de viés: (1) viés decorrente do processo de randomização; (2) enviesamento devido a desvios das intervenções pretendidas; (3) viés devido à falta de dados de resultado; (4) viés na medição dos resultados e (5) viés na seleção dos resultados relatados. As opções para responder às questões são "sim", "provavelmente sim", "provavelmente não", "não" e "sem informação". O risco de viés para cada domínio é avaliado como "baixo risco de viés", "algumas preocupações" e "alto risco de viés”36.

\section{RESULTADOS}

Foram encontrados 1270 artigos na busca preliminar (Apêndice $\mathrm{A}$ ), havendo a exclusão de 188 duplicatas. A etapa de leitura do título e do resumo com aplicação dos critérios de exclusão foi realizada com os 1082 estudos restantes, dos quais 91 foram considerados elegíveis e tiveram o conteúdo lido na íntegra. Desses, 21 estudos foram incluídos na revisão para extração e descrição dos dados.

\section{DISCUSSÃO}

Entendendo que a adesão está relacionada a múltiplos fatores que precisam ser identificados para possibilitar medidas de intervenção 37 , o presente tema é de relevância para a saúde pública, uma vez que o não alcance dos objetivos terapêuticos com o uso de anticoagulantes orais pode acarretar eventos adversos pela falha terapêutica e exacerbação do efeito anticoagulante, representados por tromboembolismo e sangramento, respectivamente, ou mesmo morte.

Assim sendo, é necessário construir pilares para apoiar o processo de assistência à saúde e produzir conhecimentos que proporcionem a elaboração de medidas para beneficiar o paciente, sendo a abordagem da não adesão ao tratamento um assunto de extrema relevância no cenário do cuidado ao paciente.

Os resultados desta revisão indicarão fatores associados à não adesão ao tratamento com derivados cumarínicos e anticoagulantes orais de ação direta. Uma análise crítica das informações coletadas será realizada e os achados podem ser relevantes para produzir conhecimento novo que possa promover a efetividade e segurança no uso do medicamento na prática clínica. Os resultados serão divulgados de forma narrativa por meio de artigos científicos, com descrição dos métodos empregados e avaliação crítica dos resultados.

\section{CONCLUSÃO}

O conhecimento dos fatores associados à não adesão à anticoagulação oral pode favorecer o planejamento de ações para melhoria assistencial ao paciente. Desse modo, espera-se que a revisão contribua para identificação desses fatores e que a abordagem dos mesmos na prática clínica resulte em melhoria na adesão e no alcance dos objetivos terapêuticos, contribuindo para implementação de um tratamento efetivo e seguro.

\section{REFERÊNCIAS}

1- Luger S, Hohmann C, Niemann D, Kraft P, Gunreben I, Neumann-Haefelin $\mathrm{T}$, et al. Adherence to oral anticoagulant therapy in secondary stroke prevention - Impact of the novel oral anticoagulants. Patient Prefer Adherence. 2015;9(ns):695-1705.

2- Doherty JU, Gluckman TJ, Hucker WJ, Januzzi JL, Ortel TL, Saxonhouse SJ, et al. ACC Expert Consensus Decision Pathway for Periprocedural Management of Anticoagulation in Patients With Nonvalvular Atrial Fibrillation: A Report of the American College of Cardiology Clinical Expert Consensus Document Task Force. J Am Coll Cardiol. 2017;69(7):871-898.

3- Beaser AD, Cifu AS. Management of Patients With Atrial Fibrillation. JAMA Clinical Guidel Synopsis. 2019;321(11):1100-1101.

4- Roth GA, Abate D, Abate KH, Abay SM, Abbafati C, Abbasi N, et al. Global, regional, and national age-sex-specific mortality for 282 causes of death in 195 countries and territories, 1980-2017: a systematic analysis for the Global Burden of Disease Study 2017. Lancet. 2018;392(10159):1736-

\section{8.}

5- Fiumara K, Goldhaber SZ. A patient's guide to taking coumadin/warfarin. Circulation. 2009;119(8):1-9.

6- Bauer KA. Pros and cons of new oral anticoagulants. Hematology Am Soc Hematol Educ Program. 2013;1(ns):464-470.

7- Howard P. New oral anticoagulants for stroke prevention in atrial fibrillation: More choices bring more challenges. Hosp Pharm. 2013;48(5):366371.

8- Connolly SJ, Ezekowitz MD, Yusuf S, Eikelboom J, Oldgren J, Parekh A, et al. Dabigatran versus warfarin in patients with atrial fibrillation. N Engl J Med. 2009;361(12):1139-1151.

9- Granger CB, Alexander JH, McMurray JJV, Lopes RD, Hylek EM, Hanna M, et al. Apixaban versus Warfarin in Patients with Atrial Fibrillation. N Engl J Med. 2011;365(11):981-992. 


\section{REFERÊNCIAS}

10- Patel MR, Mahaffey KW, Garg J, Pan G, Singer DE, Hacke W, et al. Rivaroxaban versus Warfarin in Nonvalvular Atrial Fibrillation. N Engl J Med. 2011;365(10):883-891.

11- Giugliano RP, Ruff CT, Braunwald E, Murphy SA, Wiviott SD, Halperin $\mathrm{JL}$, et al. Edoxaban versus warfarin in patients with atrial fibrillation. $\mathrm{N}$ Engl J Med. 2013;369(ns):2093-2104.

12- Deshpande CG, Kogut S, Willey C. Real-world health care costs based on medication adherence and risk of stroke and bleeding in patients treated with novel anticoagulant therapy. J Manag Care Spec Pharm. 2018;24(5):430-439.

13- Caterina R de, Ageno W, Agnelli G, Chan NC, Diener HC, Hylek E, Raskob GE, Siegal DM, Verheugt FWA, Lip GYH, Weitz JI. The Non-Vitamin K Antagonist Oral Anticoagulants in Heart Disease: Section V-Special Situations. Thromb Haemost. 2019; 119(1):14-38.

14- Kim S, Gaweda AE, Wu D, Li L, Rai SN, Brier ME. Simplified warfarin dose-response pharmacodynamic models. Biomed Eng - Appl Basis Commun. 2015;27(1):1-15.

15- Leite PM, Castilho RO, Ribeiro ALP, Martins MAP. Consumption of medicinal plants by patients with heart diseases at a pharmacist-managed anticoagulation clinic in Brazil. Int J Clin Pharm. 2016;38(2):223-227.

16- Holbrook AM, Pereira JA, Labiris R, McDonald H, Douketis JD, Crowther $M$. Systematic overview of warfarin and its drug and food interactions. Arch Intern Med [Internet]. 2005;165(10):1095-106. Available from: http://ovidsp.ovid.com/ovidweb.cgi?T=JS\&PAGE=reference\&D=emed7\&N $E W S=N \& A N=2005233410$

17- Martins MAP, Carlos PPS, Ribeiro DD, Nobre VA, César CC, Rocha MOC, et al. Warfarin drug interactions: A comparative evaluation of the lists provided by five information sources. Eur J Clin Pharmacol. 2011;67(12):1301-1308.

18- Ageno W, Gallus AS, Wittkowsky A, Crowther M, Hylek EM, Palareti G. Oral Anticoagulant Therapy. Chest. 2012;141(2):44-88.

19- Fareed J, Thethi I, Hoppensteadt D. Old versus new oral anticoagulants: Focus on pharmacology. Annu Rev Pharmacol Toxicol. 2012;52(ns):79-99.

20- Mekaj YH, Mekaj AY, Duci SB, Miftari El. New oral anticoagulants: Their advantages and disadvantages compared with vitamin $\mathrm{K}$ antagonists in the prevention and treatment of patients with thromboembolic events. Ther Clin Risk Manag. 2015;11(ns):967-977.

21- Beyer-Westendorf J, Ehlken B, Evers T. Real-world persistence and adherence to oral anticoagulation for stroke risk reduction in patients with atrial fibrillation. Europace. 2016;18(8):1150-1157.

22- Gieling E, de Vries F, Williams R, van Onzenoort HAW, de Boer A, ten Cate $\mathrm{V}$, et al. Mortality risk in atrial fibrillation: the role of aspirin, vitamin $\mathrm{K}$ and non-vitamin K antagonists. Int J Clin Pharm. 2019;41(6):1536-1544.

23- Labovitz DL, Shafner L, Reyes GM, Virmani D, Hanina A. Using Artificial Intelligence to Reduce the Risk of Nonadherence in Patients on Anticoagulation Therapy. Stroke. 2017;48(5):1416-1419.

24- American Heart Association. What are Direct-Acting Oral Anticoagulants (DOACs)? 2017; Available from: https://www.heart.org/-/media/data-import/downloadables/4/5/6/abh-what-are-doacs-ucm_494807.pdf
25- Davis NJ, Billett HH, Cohen HW, Arnsten JH. Impact of adherence, knowledge, and quality of life on anticoagulation control. Ann Pharmacother. 2005;39(4):632-636.

26- Kimmel SE. The Influence of Patient Adherence on Anticoagulation Control With Warfarin. Arch Intern Med. 2007;167(3):229.

27- Kääriäinen $M$, Paukama $M$, Kyngäs $H$. Adherence with health regimens of patients on warfarin therapy. J Clin Nurs. 2012;22(1-2):89-96.

28- World Health Organization. Adherence to Long-Term Therapies: Evidence for Action. Geneva: World Health Organization. 2003. Available from: https://www.who.int/chp/knowledge/publications/adherence_report/ en/.

29- Osterberg L, Blaschke T. Adherence to Medication. N Engl J Med. 2005;353(5):487-497.

30- Shi L, Liu J, Koleva Y, Fonseca V, Kalsekar A, Pawaskar M. Concordance of Adherence Measurement Using Self-Reported Adherence Questionnaires and Medication Monitoring Devices. Pharmacoeconomics. 2010;28(12):1097-1107.

31- Moher D, Shamseer L, Clarke $M$, Ghersi $D$, Liberati $A$, Petticrew $M$, Shekelle P, Stewart LA. Preferred Reporting Items for Systematic Review and Meta-Analysis Protocols (PRISMA-P) 2015 statement. Syst Rev. 2015;4(1):1. doi: 10.1186/2046-4053-4-1

32- Ouzzani M, Hammady H, Fedorowicz Z, Elmagarmid A. Rayyan a web and mobile app for systematic reviews. Systematic Reviews. 2016:5:210

33- Page MJ, McKenzie JE, Bossuyt PM, Boutron I, Hoffmann TC, Mulrow CD, et al. The PRISMA 2020 statement: an updated guideline for reporting systematic reviews.

34- Wells GA, Shea B, O'Connell D, Peterson J, Welch V, Losos M, P. Tugwell. The Newcastle-Ottawa Scale (NOS) for assessing the quality of non-randomised studies in meta-analyses, 2014. Available from: http://www.ohri. ca/programs/clinical_epid

emiology/oxford.asp

35- Ma LL, Wang YY, Yang ZH, Huang D, Hong W, Zeng XT. Methodological quality (risk of bias) assessment tools for primary and secondary medical studies: what are they and which is better?. Military Med Res. 2020;7(7):111.

36- Sterne JAC, Savović J, Page MJ, Elbers RG, Blencowe NS, Boutron I, et al. RoB 2: a revised tool for assessing risk of bias in randomised trials. BMJ (Clinical Research ed.). 2019;366(ns):1-8.

37- Alves EG, Martins NC, Santos RM, Miranda da Silva SS, Ferreira, SDRS. Adesão ao tratamento de usuários hipertensos assistidos por uma equipe de estratégia da saúde da família. Saud Coletiv. (Barueri) [Internet].2021;(11)N.65. 


\section{artigo}

Sousa, W. J. F. N., Guimarães, N. S., Viana C. C., Medeiros, A. F., Vianna, M. S.

Fatores associados à não adesão ao uso de anticoagulantes orais: protocolo de revisão sistemática

Apêndice A: Estratégias de busca e registro de estudos em abril de 2021, sem filtro por idioma ou data de publicação

MEDLINE

EMBASE

CINAHL (EBSCO)

LILACS

\section{Estratégia de pesquisa}

Registros recuperados

\#1 Adult [Mesh Terms] OR (Adults) OR Aged [Mesh] OR (Elderly) \#2 Coumarins [Mesh Terms] OR (Coumarines) OR (1,2-Benzopyrone Derviatives) OR (1,2 Benzopyrone Derviatives) OR (Derviatives, 1,2-Benzopyrone) OR (Benzopyran-2-ones) OR (Benzopyran 2 ones) OR (Coumarin Derivatives) OR (Derivatives, Coumarin) OR (1,2-Benzopyrones) OR (1,2 Benzopyrones) OR (1,2-Benzo-Pyrones) OR (1,2 Benzo Pyrones) OR "Factor Xa Inhibitors" [Mesh Terms] OR (Direct Factor Xa Inhibitors) OR (Direct-Acting Oral Anticoagulants) OR (Anticoagulants, Direct-Acting Oral) OR (Direct Acting Oral) OR (Anticoagulants) OR (Oral Anticoagulants, Direct-Acting) \#3 "Medication Adherence" [Mesh Terms] OR (Medication Non-Adherence) OR (Medication Non Adherence) OR (Non-Adherence, Medication) OR (Medication Nonadherence) OR (Medication Non-Compliance) OR (Medication Non Compliance) OR (Non-Compliance, Medication) \#1 AND \#2 AND \#3

\#1 'Adult'/syn OR (Adult/exp) OR Aged/syn OR (Aged/exp) AND [embase]/lim \#2 'coumarin derivative'/syn OR ('coumarin derivative'/exp) OR 'blood clotting factor 10a inhibitor'/syn OR ('blood clotting factor 10a inhibitor'/exp) AND [embase]/lim \#3 'medication compliance'/syn OR ('medication compliance'/exp) AND [embase]/lim \#1 AND \#2 AND \#3

(Adult [Mesh Terms] OR (Adults) OR Aged [Mesh] OR (Elderly) ) AND ( Coumarins [Mesh Terms] OR (Coumarines) OR (1,2-Benzopyrone Derviatives) OR (1,2 Benzopyrone Derviatives) OR (Derviatives, 1,2-Benzopyrone) OR (Benzopyran-2-ones) OR (Benzopyran 2 ones) OR (Coumarin Derivatives) OR (Derivatives, Coumarin) OR (1,2-Benzopyrones) OR (1,2 Benzopyrones) OR (1,2-Benzo-Pyrones) OR (1,2 Benzo Pyrones) OR "Factor Xa Inhibitors" [Mesh Terms] OR (Direct Factor Xa Inhibitors) OR (Direct-Acting Oral Anticoagulants) OR (Anticoagulants, Direct-Acting Oral) OR (Direct Acting Oral) OR (Anticoagulants) OR (Oral Anticoagulants, Direct-Acting) ) AND ( "Medication Adherence" [Mesh Terms] OR (Medication Non-Adherence) OR (Medication Non Adherence) OR (Non-Adherence, Medication) OR (Medication Nonadherence) OR (Medication Non-Compliance) OR (Medication Non Compliance) OR (Non-Compliance, Medication) )

\#1 "Adult" OR "Adults" OR mh:"Idoso" OR "Aged" OR "Anciano"

\#2 "Cumarínicos" OR "Coumarins" OR "Cumarinas" OR "1,2-Benzopironas" OR "Benzopiran-2-Onas" OR "Cumarinas" OR

"Inibidores do Fator Xa" OR "Factor Xa Inhibitors" OR "Inhibidores del Factor Xa" OR "Anticoagulantes Orais de Ação Direta" OR "Inibidor do Fator Xa" OR "Inibidores Diretos do Fator Xa" \#3 "Adesão à Medicação" OR "Medication Adherence" OR "Cumplimiento de la Medicación" OR "Não Aderência ao Medicamento" OR "Não Adesão ao Medicamento Submissão ao Medicamento" \#1 AND \#2 AND \#3 
Pesquisa: ((Adult [Mesh Terms] OR (Adults) OR Aged [Mesh] OR (Elderly)) AND (Coumarins [Mesh Terms] OR (Coumarines) OR (1,2-Benzopyrone Derviatives) OR (1,2 Benzopyrone Derviatives) OR (Derviatives, 1,2-Benzopyrone) OR (Benzopyran-2-ones) OR (Benzopyran 2 ones) OR (Coumarin Derivatives) OR (Derivatives, Coumarin) OR (1,2-Benzopyrones) OR (1,2 Benzopyrones) OR (1,2-Benzo-Pyrones) OR (1,2 Benzo Pyrones) OR "Factor Xa Inhibitors" [Mesh Terms] OR (Direct Factor Xa Inhibitors) OR (Direct-Acting Oral Anticoagulants) OR (Anticoagulants, Direct-Acting Oral) OR (Direct Acting Oral) OR (Anticoagulants) OR (Oral Anticoagulants, Direct-Acting))) AND ("Medication Adherence" [Mesh Terms] OR (Medication Non-Adherence) OR (Medication Non Adherence) OR (Non-Adherence, Medication) OR (Medication Nonadherence) OR (Medication Non-Compliance) OR (Medication Non Compliance) OR (Non-Compliance, Medication)) ("adult"[MeSH Terms] OR ("adult"[MeSH Terms] OR "adult"[All Fields] OR "adults"[All Fields] OR "adult s"[All Fields]) OR "aged"[MeSH Terms] OR ("aged"[MeSH Terms] OR "aged"[All Fields] OR "elderly"[All Fields] OR "elderlies"[All Fields] OR "elderly s"[All Fields] OR "elderlys"[All Fields])) AND ("coumarins"[MeSH Terms] OR ("coumarin"[Supplementary Concept] OR "coumarin"[All Fields] OR "coumarinic"[All Fields] OR "coumarins"[MeSH Terms] OR "coumarins"[All Fields] OR "coumarine"[All Fields] OR "coumarines"[All Fields]) OR ("coumarins"[MeSH Terms] OR "coumarins"[All Fields] OR ("1 2"[All Fields] AND "benzopyrone"[All Fields] AND "derviatives"[All Fields])) OR ("coumarins"[MeSH Terms] OR "coumarins"[All Fields] OR ("1 2"[All Fields] AND "benzopyrone"[All Fields] AND "derviatives"[All Fields])) OR ("coumarins"[MeSH Terms] OR "coumarins"[All Fields] OR ("derviatives"[All Fields] AND "1 2"[All Fields] AND "benzopyrone"[All Fields])) OR ("coumarins"[MeSH Terms] OR "coumarins"[All Fields] OR "benzopyran 2 ones"[All Fields]) OR ("coumarins"[MeSH Terms] OR "coumarins"[All Fields] OR "benzopyran 2 ones"[All Fields]) OR ("coumarins"[MeSH Terms] OR "coumarins"[All Fields] OR ("coumarin"[All Fields] AND "derivatives"[All Fields]) OR "coumarin derivatives"[All Fields]) OR ("coumarins"[MeSH Terms] OR "coumarins"[All Fields] OR ("derivatives"[All Fields] AND "coumarin"[All Fields]) OR "derivatives coumarin"[All Fields]) OR ("coumarins"[MeSH Terms] OR "coumarins"[All Fields] OR ("1 2"[All Fields] AND "benzopyrones"[All Fields]) OR "1 2 benzopyrones"[All Fields]) OR ("coumarins"[MeSH Terms] OR "coumarins"[All Fields] OR ("1 2"[All Fields] AND "benzopyrones"[All Fields]) OR "1 2 benzopyrones"[All Fields]) OR ("coumarins"[MeSH Terms] OR "coumarins"[All Fields] OR ("1 2"[All Fields] AND "benzo"[All Fields] AND "pyrones"[All Fields])) OR ("coumarins"[MeSH Terms] OR "coumarins"[All Fields] OR ("1 2"[All Fields] AND "benzo"[All Fields] AND "pyrones"[All Fields])) OR "Factor Xa Inhibitors"[MeSH Terms] OR ("Factor Xa Inhibitors"[Pharmacological Action] OR "Factor Xa Inhibitors"[MeSH Terms] OR ("factor"[All Fields] AND "xa"[All Fields] AND "inhibitors"[All Fields]) OR "Factor Xa Inhibitors"[All Fields] OR ("direct"[All Fields] AND "factor"[All Fields] AND "xa"[All Fields] AND "inhibitors"[All Fields]) OR "direct factor xa inhibitors"[All Fields]) OR ("Factor Xa Inhibitors"[Pharmacological Action] OR "Factor Xa Inhibitors"[MeSH Terms] OR ("factor"[All Fields] AND "xa"[All Fields] AND "inhibitors"[All Fields]) OR "Factor Xa Inhibitors"[All Fields] OR ("direct"[All Fields] AND "acting"[All Fields] AND "oral"[All Fields] AND "anticoagulants"[All Fields]) OR "direct acting oral anticoagulants"[All Fields]) OR ("Factor Xa Inhibitors"[Pharmacological Action] OR "Factor Xa Inhibitors"[MeSH Terms] OR ("factor"[All Fields] AND "xa"[All Fields] AND "inhibitors"[All Fields]) OR "Factor Xa Inhibitors"[All Fields] OR ("anticoagulants"[All Fields] AND "direct"[All Fields] AND "acting"[All Fields] AND "oral"[All Fields])) OR (("direct"[All Fields] OR "directed"[All Fields] OR "directing"[All Fields] OR "direction"[All Fields] OR "directional"[All Fields] OR "directions"[All Fields] OR "directivities"[All Fields] OR "directivity"[All Fields] OR "directs"[All Fields]) AND ("acted"[All Fields] OR "acting"[All Fields]) AND ("mouth"[MeSH Terms] OR "mouth"[All Fields] OR "oral"[All Fields])) OR ("anticoagulants"[Pharmacological Action] OR "anticoagulants"[MeSH Terms] OR "anticoagulants"[All Fields] OR "anticoagulant"[All Fields] OR "anticoagulate"[All Fields] OR "anticoagulated"[All Fields] OR "anticoagulating"[All Fields] OR "anticoagulation"[All Fields] OR "anticoagulations"[All Fields] OR "anticoagulative"[All Fields]) OR ("Factor Xa Inhibitors"[Pharmacological Action] OR "Factor Xa Inhibitors"[MeSH Terms] OR ("factor"[All Fields] AND "xa"[All Fields] AND "inhibitors"[All Fields]) OR "Factor Xa Inhibitors"[All Fields] OR ("oral"[All Fields] AND "anticoagulants"[All Fields] AND "direct"[All Fields] AND "acting"[All Fields]))) AND ("Medication Adherence"[MeSH Terms] OR ("Medication Adherence"[MeSH Terms] OR ("medication"[All Fields] AND "adherence"[All Fields]) OR "Medication Adherence"[All Fields] OR ("medication"[All Fields] AND "non"[All Fields] AND "adherence"[All Fields]) OR "medication non adherence"[All Fields]) OR ("Medication Adherence"[MeSH Terms] OR ("medication"[All Fields] AND "adherence"[All Fields]) OR "Medication Adherence"[All Fields] OR ("medication"[All Fields] AND "non"[All Fields] AND "adherence"[All Fields]) OR "medication non adherence"[All Fields]) OR ("Medication Adherence"[MeSH Terms] OR ("medication"[All Fields] AND "adherence"[All Fields]) OR "Medication Adherence"[All Fields] OR ("non"[All Fields] AND "adherence"[All Fields] AND "medication"[All Fields]) OR "non adherence medication"[All Fields]) OR ("Medication Adherence"[MeSH Terms] OR ("medication"[All Fields] AND "adherence"[All Fields]) OR "Medication Adherence"[All Fields] OR ("medication"[All Fields] AND "nonadherence"[All Fields]) OR "medication nonadherence"[All Fields]) OR ("Medication Adherence"[MeSH Terms] OR ("medication"[All Fields] AND "adherence"[All Fields]) OR "Medication Adherence"[All Fields] OR ("medication"[All Fields] AND "non"[All Fields] AND "compliance"[All Fields]) OR "medication non compliance"[All Fields]) OR ("Medication Adherence"[MeSH Terms] OR ("medication"[All Fields] AND "adherence"[All Fields]) OR "Medication Adherence"[All Fields] OR ("medication"[All Fields] AND "non"[All Fields] AND "compliance"[All Fields]) OR "medication non compliance"[All Fields]) OR ("Medication Adherence"[MeSH Terms] OR ("medication"[All Fields] AND "adherence"[All Fields]) OR "Medication Adherence"[All Fields] OR ("non"[All Fields] AND "compliance"[All Fields] AND "medication"[All Fields]) OR "non compliance medication"[All Fields])) 


\section{artigo}

Sousa, W. J. F. N., Guimarães, N. S., Viana C. C., Medeiros, A. F., Vianna, M. S.

Fatores associados à não adesão ao uso de anticoagulantes orais: protocolo de revisão sistemática

\section{Apêndice C: Modelo de fluxograma para revisões sistemáticas PRISMA 2020}

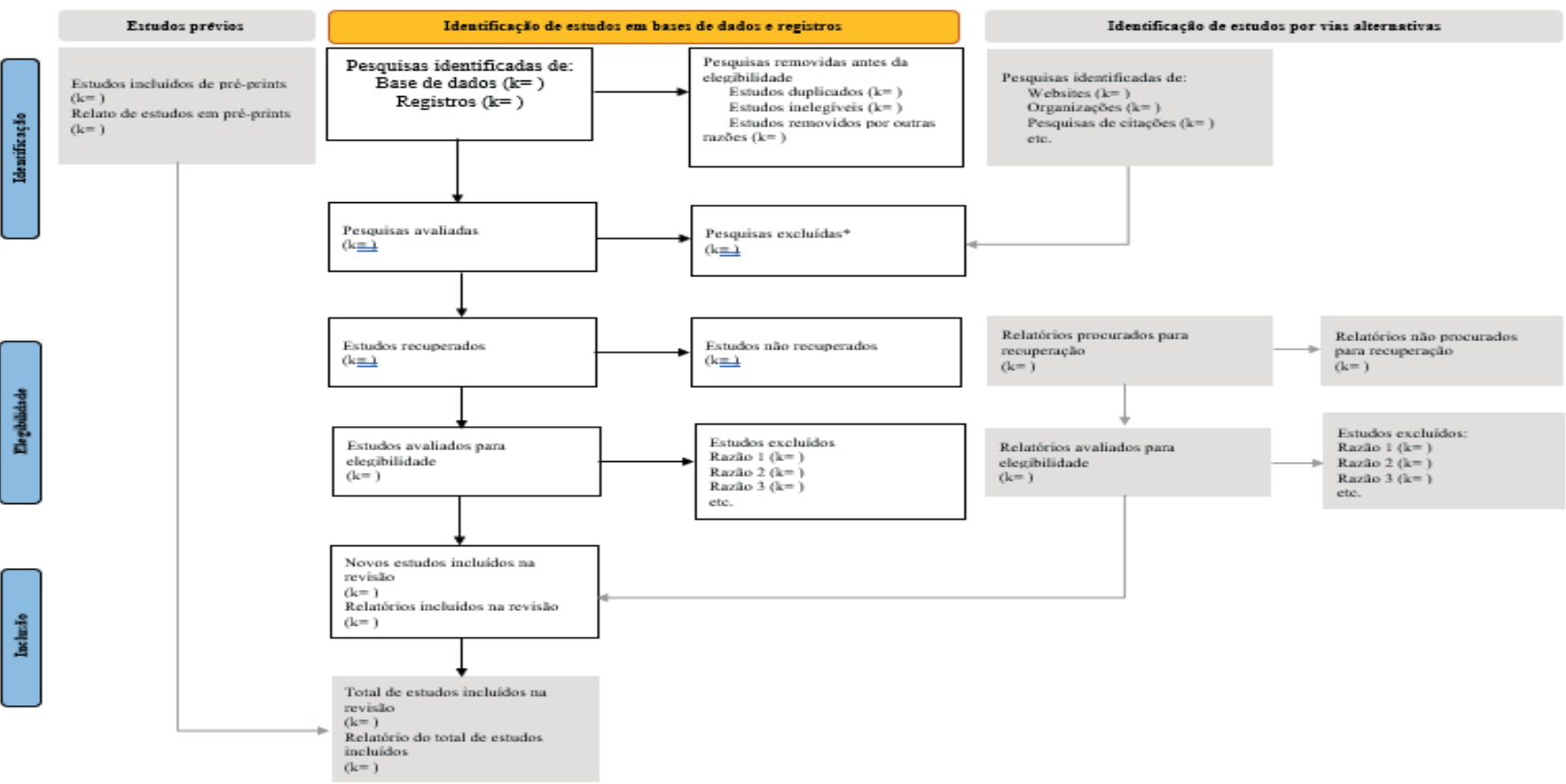

\section{Apêndice D: Tabela de extração dos dados}

\section{Informações do estudo}

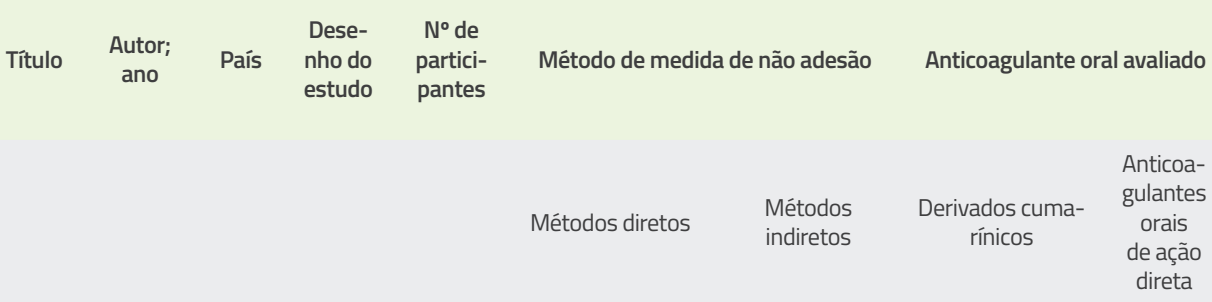

\section{Apêndice D: Tabela de extração dos dados - Continuação}

Dados sociodemográficos e comportamentais

Idade

(mé- Sexo masculino

dia $\pm \quad(n ; \%)$

Escolaridade $(\mathbf{n} ; \%)$

Consumo de bebidas alcoólicas

Tabagismo

DP*)

\section{Apêndice D: Tabela de extração dos dados - Continuação}

Dados sociodemográficos e comportamentais

Indicação da anticoagulação oral
Comorbidades

Ocorrência de eventos

Outros medicamentos em uso crônico 